\title{
PRODUÇÃO DE ETANOL CELULÓSICO POR SACARIFICAÇÃO E FERMENTAÇÃO SIMULTÂNEAS DA BIOMASSA DE PALMA FORRAGEIRA PRÉ-TRATADA EM MEIO ALCALINO
}

\author{
P. F. de SOUZA FILHO ${ }^{1}$, S. D. de OLIVEIRA Jr ${ }^{1}$, V. T. RIBEIRO ${ }^{1}$, E. S. dos SANTOS $^{1}$ e G. R. \\ $\mathrm{MACEDO}^{1}$ \\ ${ }^{1}$ Universidade Federal do Rio Grande do Norte, Departamento de Engenharia Química \\ E-mail para contato: pedrofsf@yahoo.com.br
}

\begin{abstract}
RESUMO - O uso da biomassa lignocelulósica como substrato para produção de etanol atende às necessidades atuais de se buscar novas fontes de energia que não compitam com a produção de alimentos e reduzam o impacto ambiental. Este estudo avaliou o comportamento de duas espécies de palma forrageira encontradas no Nordeste do Brasil (Opuntia fícus indica e Nopalea cochenillifera) submetidas a pré-tratamento com hidróxido de sódio como matérias-primas para produção de etanol pelo processo de sacarificação e fermentação simultâneas (SFS) usando duas cepas de Saccharomyces cerevisiae (PE-2 e CA-11). Usando a metodologia do planejamento fatorial $2^{2}$ mais 3 pontos centrais, foi estudada a influência da temperatura $\left(30,40\right.$ e $\left.45{ }^{\circ} \mathrm{C}\right)$ e da carga inicial de celulose (3, 4 e 5\%). Os ensaios mostraram maior rendimento da conversão de celulose em etanol pela cepa PE-2 usando a Nopalea $(93,81 \%)$ no ponto central. A espécie Opuntia também obteve rendimento máximo $(62,48 \%)$ no ponto central usando a cepa PE-2.
\end{abstract}

\section{INTRODUÇÃO}

Dentre as muitas culturas alternativas para a produção de álcool etílico, pode-se citar a palma forrageira, que tem despertado o interesse de muitos pesquisadores, devido a sua vasta disponibilidade, ausência de entressafra, baixa necessidade de tecnologia para cultivo, além de ser uma cultura bastante difundida, altamente resistente à seca e de fácil plantio (Torres Neto, 2010). Na região Nordeste do Brasil, são encontrados três tipos principais de palma: a gigante, da espécie Opuntia fícus indica; a redonda, Opuntia sp; e a miúda, Nopalea cochenilifera (Silva e Santos, 2006). Diversos trabalhos têm mostrado bons resultados com o uso da palma gigante para produção de etanol celulósico (Baracho et al., 2009; Kuloyo, 2011; Torres Neto, 2010).

O processo de sacarificação e fermentação simultâneas (SFS) foi proposto como forma de tornar economicamente atrativo o etanol celulósico, visto que nesta estrutura, as celulases catalisam a hidrólise da celulose em glicose, que é simultaneamente fermentada em álcool em um único reator. Esta estratégia reduz o potencial de contaminação microbiológica, diminui os custos de equipamento e previne a ação enzimática de ser inibida pelos açúcares produzidos, aumentando assim o rendimento desta etapa (Fox et al., 2012). 


\section{9 a 22 de outubro de 2014 \\ Florianópolis/SC}

Este trabalho teve como objetivo realizar um estudo sobre a viabilidade técnica do uso dos cladódios de duas espécies de palma forrageira como substrato para produção de etanol celulósico utilizando um processo de sacarificação e fermentação simultâneas, analisando-se o efeito que a concentração inicial de celulose e a temperatura exercem sobre a produção de etanol por duas diferentes cepas de Saccharomyces cerevisiae.

\section{MATERIAL E MÉTODOS}

\subsection{Palma Forrageira}

Os cladódios de palma forrageira das duas espécies testadas neste trabalho ( $N$. cochenillifera e O. ficus indica) foram obtidos na fazenda Aquafrutas II, localizada no município de Touros, Rio Grande do Norte, sendo estas de uma variedade sem espinhos encontrada comumente no interior do Rio Grande do Norte. Após a seleção e lavagem, os cladódios foram cortados em pedaços de aproximadamente $3 \mathrm{~cm} \times 3 \mathrm{~cm} \times 1 \mathrm{~cm}$ e secos. $\mathrm{O}$ material foi então triturado em moinho de facas tipo willey e a fração de finos passantes pela peneira de 20 mesh $(0,841 \mathrm{~mm})$ foi coletada. O material passou então por um processo de sucessivas lavagens com água quente (aproximadamente $70{ }^{\circ} \mathrm{C}$ ) para remoção de materiais solúveis, que poderiam diminuir a eficiência do pré-tratamento, sendo posteriormente seco novamente. O material foi armazenado em recipiente fechado e guardado em local seco a temperatura ambiente para evitar a sua degradação.

\subsection{Pré-Tratamento}

O procedimento consistiu em levar uma mistura contendo $10 \%(\mathrm{p} / \mathrm{v})$ de material lignocelulósico em solução de $\mathrm{NaOH} 0,5 \%$ (p/v) à autoclave, onde era mantida por 30 min a $1,05 \mathrm{~atm}\left(121{ }^{\circ} \mathrm{C}\right)$. Ao fim do pré-tratamento, o material lignocelulósico era submetido a sucessivas lavagens com água da torneira (perfazendo cerca de $10 \% \mathrm{p} / \mathrm{v}$ de biomassa em cada lavagem) a fim de remover os compostos recalcitrantes formados pela degradação da lignina. A lavagem prosseguia até que o $\mathrm{pH}$ do filtrado se aproximasse do valor da água da torneira e sua cor desaparecesse.

A biomassa foi caracterizada com relação ao seu teor de extrativos (Pitarelo, 2007), polissacarídeos (celulose e hemicelulose) e lignina (Sluiter et al., 2011) e cinzas (Vasconcelos, 2012) conforme os procedimentos descritos na literatura.

\subsection{Microrganismos e Preparo do Inóculo}

Uma amostra de levedura $S$. cerevisiae da cepa LNF CA-11 foi gentilmente cedida pela LNF Latino Americana. A cepa PE-2 (citada por Basso et al., 2008) utilizada neste trabalho foi obtida do estoque de culturas do Laboratório de Engenharia Bioquímica do Departamento de Engenharia Química da Universidade Federal do Rio Grande do Norte. Estes microrganismos foram mantidos em placa de Petri em meio de ágar YEPD (Ágar-ágar $20 \mathrm{~g} / \mathrm{L}$; Extrato de Levedura $10 \mathrm{~g} / \mathrm{L}$; Peptona 20 $\mathrm{g} / \mathrm{L}$; Glicose $20 \mathrm{~g} / \mathrm{L})$ com $\mathrm{pH}$ corrigido para 5,0 $\left(\mathrm{H}_{2} \mathrm{SO}_{4}\right.$ 0,1 M). Após inoculação, as placas foram deixadas por $48 \mathrm{~h}$ a $28-30{ }^{\circ} \mathrm{C}$ para crescimento das colônias e, posteriormente, guardadas sob refrigeração $\left(4^{\circ} \mathrm{C}\right)$ seguindo a metodologia de Ruiz et al. (2012). 


\section{9 a 22 de outubro de 2014 \\ Florianópolis/SC}

Para o preparo do pré-inóculo, $100 \mathrm{~mL}$ de uma solução contendo glicose $(30,00 \mathrm{~g} / \mathrm{L})$, extrato de levedura $(5,00 \mathrm{~g} / \mathrm{L}),\left(\mathrm{NH}_{4}\right)_{2} \mathrm{SO}_{4}(10,00 \mathrm{~g} / \mathrm{L}), \mathrm{KH}_{2} \mathrm{PO}_{4}(4,50 \mathrm{~g} / \mathrm{L}), \mathrm{MgSO}_{4} .7 \mathrm{H}_{2} \mathrm{O}(1,00 \mathrm{~g} / \mathrm{L}), \mathrm{ZnSO}_{4}$ $(0,65 \mathrm{~g} / \mathrm{L})$ e $\mathrm{pH}$ corrigido para $5,0\left(\mathrm{H}_{2} \mathrm{SO}_{4}\right.$ 0,1 M) foram inoculados em frascos Erlenmeyer de 250 $\mathrm{mL}$ mantidos a $35^{\circ} \mathrm{C}$ e $150 \mathrm{rpm}$ por 22 horas. Neste momento, uma alíquota de $10 \mathrm{~mL}$ era transferida para outro Erlenmeyer contendo $90 \mathrm{~mL}$ de solução com mesma composição inicial do pré-inóculo, sendo disposto nas mesmas condições. Após 9 horas de crescimento, a solução atingia novamente valor máximo de concentração celular e uma alíquota era transferida para o Erlenmeyer onde ocorreria a sacarificação e fermentação simultâneas de modo que a concentração inicial de microrganismos desejada fosse obtida.

\subsection{Sacarificação e Fermentação Simultâneas (SFS)}

Os ensaios de sacarificação e fermentação simultâneas foram realizados em frascos Erlenmeyers de $250 \mathrm{~mL}$, em mesa agitadora com agitação e temperatura controladas. Como parâmetros iniciais, utilizou-se uma carga enzimática de $20 \mathrm{FPU} / \mathrm{g}$ de biomassa, concentração inicial de microrganismos de $4 \mathrm{~g} / \mathrm{L}$, agitação de $150 \mathrm{rpm}$ e tempo total de 48 horas. Para complementar as necessidades nutricionais das leveduras, foi adicionado ao meio fermentativo contendo a palma uma solução contendo $4 \mathrm{~g} / \mathrm{L}$ de extrato de levedura, $2 \mathrm{~g} / \mathrm{L}$ de $\left(\mathrm{NH}_{4}\right)_{2} \mathrm{SO}_{4}, 2 \mathrm{~g} / \mathrm{L}$ de $\mathrm{KH}_{2} \mathrm{PO}_{4}$ e $0,75 \mathrm{~g} / \mathrm{L}$ de $\mathrm{MgSO}_{4} \cdot 7 \mathrm{H}_{2} \mathrm{O}$. O volume desta solução a ser adicionada era determinado pela quantidade de inóculo previamente adicionado. O coquetel enzimático ACCELLERASE ${ }^{\circledR} 1500$, gentilmente cedido pela GENENCOR $^{\circledR}$, foi utilizado neste trabalho.

As amostras obtidas foram levadas para análise por Cromatografia Líquida de Alta Eficiência (CLAE) a fim de determinar a concentração de etanol ao fim da fermentação. Avaliou-se o rendimento de etanol dos sistemas experimentais nas temperaturas de 35,40 e $45^{\circ} \mathrm{C}$ (níveis $-1,0$ e 1 , respectivamente) e nas concentrações iniciais de substrato de 3,4 e $5 \%(\mathrm{p} / \mathrm{v}$ ) de celulose (níveis $-1,0$ e 1 , respectivamente), tendo sido efetuado um estudo fatorial $2^{2}$ com triplicata no ponto central. O rendimento em etanol da SFS ou a conversão de celulose foi calculado de acordo com a Equação 1 (Dowe \& McMillan, 2008), que relaciona a diferença entre a concentração inicial e final de etanol com a concentração inicial de celulose na mistura, usando os fatores estequiométricos de conversão de celulose em glicose e de glicose em etanol:

$$
\% \text { Rendimento }=\frac{[\mathrm{EtOH}]_{\mathrm{f}}-[\mathrm{EtOH}]_{0}}{0,51 \times\left(1,111 \times[\text { Celulose }]_{0}\right)} \times 100 \%
$$

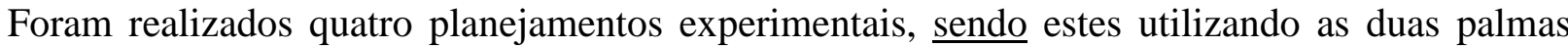
forrageiras e usando as leveduras $S$. cerevisiae PE-2 e CA-11. O programa computacional STATISTICA@ (versão 7.0) foi utilizado para obtenção dos efeitos principais das variáveis e de suas interações, dos dados de análise de variância e para a obtenção de superfícies de resposta.

\section{RESULTADOS E DISCUSSÃO}

\subsection{Caracterização da Biomassa}


Os valores obtidos pela caracterização da biomassa são apresentados na Tabela 1.

Tabela 1 - Caracterização da biomassa de palma forrageira

\begin{tabular}{ccc}
\hline Componentes & N. cochenillifera & O. ficus indica \\
\hline Celulose (\%) & $43,19 \pm 4,05$ & $31,99 \pm 0,10$ \\
Hemicelulose (\%) & $22,38 \pm 0,58$ & $19,59 \pm 0,45$ \\
Lignina (\%) & $15,96 \pm 0,37$ & $20,49 \pm 0,04$ \\
Extrativos (\%) & $3,36 \pm 2,10$ & $13,09 \pm 1,95$ \\
Cinzas (\%) & $6,28 \pm 0,25$ & $5,71 \pm 0,07$ \\
Total (\%) & 91,17 & 90,87 \\
\hline
\end{tabular}

A palma $N$. cochenillifera apresentou maior teor de polissacarídeos, tanto celulose quanto hemicelulose, e teor de lignina inferior que a $O$. fícus indica. O baixo teor de lignina das amostras em relação às biomassas mais estudadas, como o bagaço de cana-de-açúcar, que é da ordem de $26,4 \%$ (Rabelo et al., 2011) chama a atenção, pois este é um dos principais fatores que limita a extensão da hidrólise enzimática e ainda contribui para a liberação de materiais recalcitrantes, diminuindo o rendimento da fermentação alcoólica.

Malainine et al. (2003) trabalharam com amostras de $O$. fícus indica com teor de lignina de apenas 3,6\% e, para a mesma espécie, Kuloyo (2012) encontrou o valor de 7,9\%. Torres Neto (2010) identificou um teor de lignina de $4,77 \%$ também para a palma gigante (do gênero Opuntia). Deve ser destacado que um determinado material celulósico apresenta variação em sua composição dependendo das condições de cultivo, clima, idade e parte da planta, o que pode explicar a diferença entre os valores obtidos neste trabalho e aqueles encontrados na literatura.

\subsection{Ensaios de Sacarificação e Fermentação Simultâneas}

3.2.1. Cepa LNF CA-11: A Tabela 2 apresenta os resultados obtidos dos planejamentos experimentais com as espécies $N$. cochenillifera e $O$. fícus indica submetidas ao pré-tratamento alcalino como substrato e tendo como agente de fermentação a levedura $S$. cerevisiae, cepa LNF CA11, que possui alto poder de floculação.

Tabela 2 - Planejamento fatorial $\left(2^{2}\right)$ e resultados do rendimento de conversão de celulose em etanol pela cepa LNF CA-11 usando palma forrageira pré-tratada via rota alcalina como substrato.

\begin{tabular}{rcccc}
\hline \multirow{2}{*}{ Ensaio } & \multirow{2}{*}{ Temperatura } & \multirow{2}{*}{ Carga inicial de celulose } & \multicolumn{2}{c}{ Rendimento em etanol (\%) } \\
\cline { 3 - 5 } & & & N. cochenillifera & O. fícus indica \\
\hline 1 & -1 & -1 & 75,93 & 31,01 \\
2 & +1 & -1 & 11,61 & 11,42 \\
3 & -1 & +1 & 68,02 & 35,58 \\
4 & +1 & +1 & 9,64 & 8,01 \\
5 & 0 & 0 & 29,67 & 29,06 \\
6 & 0 & 0 & 33,05 & 26,11 \\
7 & 0 & 0 & 27,95 & 27,59 \\
\hline
\end{tabular}




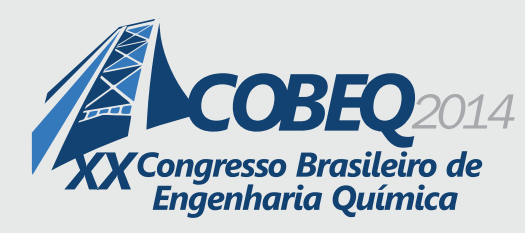

19 a 22 de outubro de 2014
Florianópolis/SC

Os resultados podem ser visualizados nas superfícies de resposta (Figuras 1A e 1B) e nos diagramas de Pareto (Figuras 1C e 1D). O efeito da carga inicial de celulose demonstrou-se não significativa (nível de confiança de 95\%) dentro dos valores estudados, sugerindo que uma carga mais elevada de celulose inicial pode ser utilizada sem o comprometimento do rendimento da conversão da celulose, gerando assim uma economia no volume de meio nutritivo adicionado à biomassa. A interação entre a temperatura e a carga inicial de biomassa também se demonstrou não significativa dentro do mesmo nível de confiança.
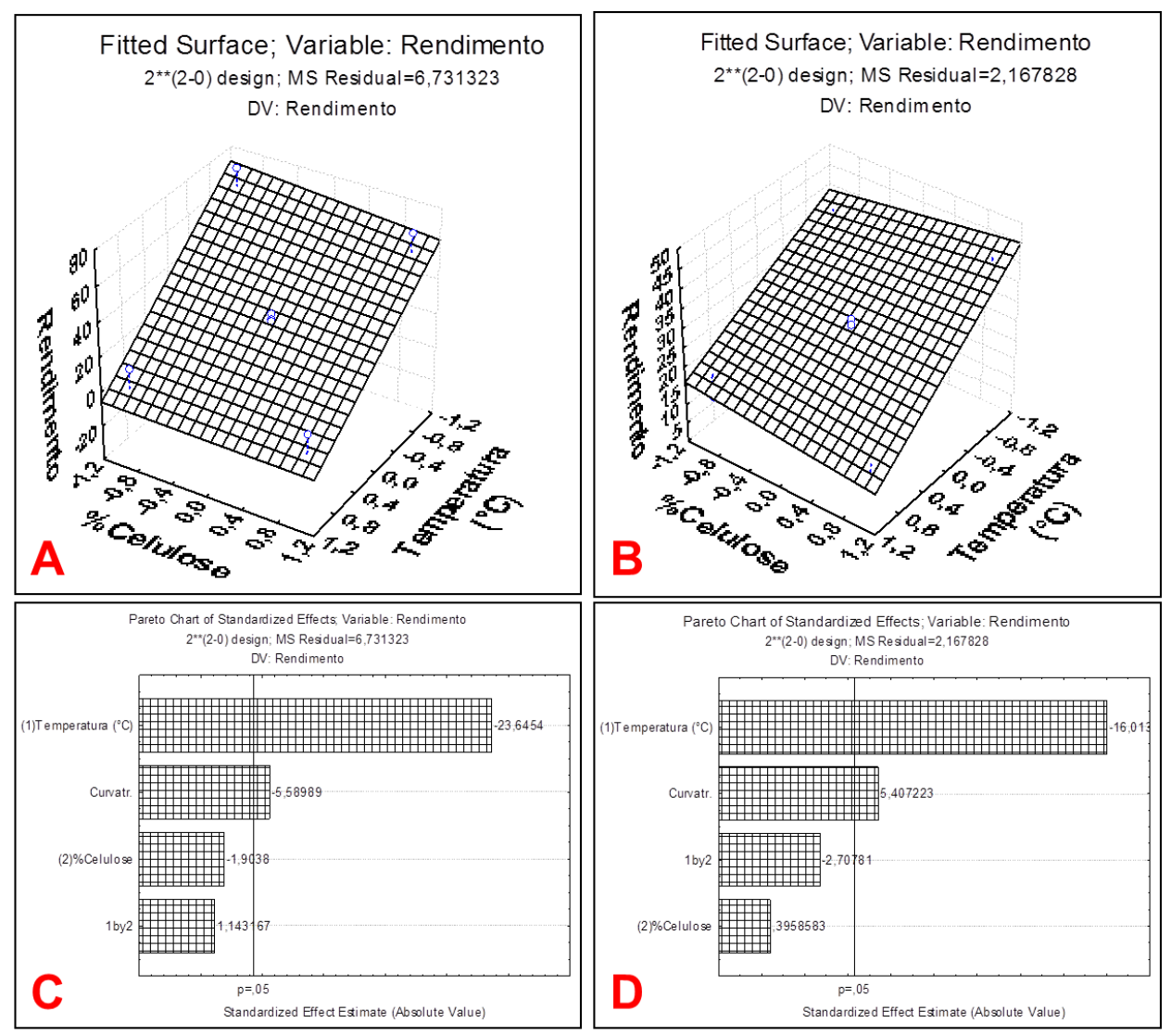

Figura 1 - Curvas de superfície de resposta do processo SFS usando o microrganismo $S$. cerevisiae, cepa LNF CA-11, e biomassa de $N$. cochenillifera (A) e $O$. ficus indica (B) como substrato e diagramas de Pareto usando $N$. cochenillifera $(\mathrm{C})$ e $O$. ficus indica (D). Rendimento da conversão de celulose em etanol em função da temperatura e da carga inicial de celulose.

Foi avaliado na análise de variância o termo de curvatura. Este termo possui significância estatística, os dados são mais bem ajustados por um modelo não linear. Como observado no Diagrama de Pareto, a curvatura apresentou significância estatística para os dados obtidos em ambos os casos. Um modelo linear, portanto, não representa os dados de forma satisfatória.

Pode-se observar que o rendimento comportou-se de forma inversamente proporcional em relação à temperatura, tendo sido observado valor máximo de rendimento no nível inferior de temperatura $\left(35^{\circ} \mathrm{C}\right)$. Este resultado sugere que, apesar da atividade enzimática ser favorecida com o 
aumento da temperatura, o microrganismo não se comportou bem num ambiente mais quente. $\mathrm{O}$ efeito da temperatura foi significativo dentro do nível de confiança de $95 \%$.

O resultado obtido apresenta comportamento oposto ao apresentado por Ruiz et al. (2012), no qual a mesma cepa apresentou efeito positivo com o aumento da temperatura na faixa de 30 a $45^{\circ} \mathrm{C}$.

Os valores máximos de rendimento para a Opuntia ficaram bem abaixo dos obtidos para a Nopalea. Enquanto que a $N$. cochenilifera apresentou rendimentos superiores a 70\%, a $\mathrm{O}$. ficus indica atingiu valor máximo de apenas $35,58 \%$.

3.2.2. Cepa PE-2: A Tabela 3 apresenta os resultados obtidos dos planejamentos experimentais $2^{2}$ com triplicata no ponto central com as espécies $N$. cochenilifera e $O$. ficus indica submetidas ao pré-tratamento alcalino como substrato e tendo como agente de fermentação a levedura $S$. cerevisiae, cepa PE-2.

Tabela 3 - Planejamento fatorial $\left(2^{2}\right)$ e resultados do rendimento de conversão de celulose em etanol pela cepa PE-2 usando palma forrageira pré-tratada via rota alcalina como substrato.

\begin{tabular}{ccccc}
\hline \multirow{2}{*}{ Ensaio } & \multirow{2}{*}{ Temperatura } & $\begin{array}{c}\text { Carga inicial de } \\
\text { celulose }\end{array}$ & \multicolumn{2}{c}{ Rendimento em etanol (\%) } \\
\cline { 4 - 5 } & & -1 & N. cochenilifera & O. ficus indica \\
\hline 1 & -1 & -1 & 63,50 & 44,37 \\
2 & +1 & +1 & 13,04 & 22,86 \\
3 & -1 & +1 & 8,99 & 32,89 \\
4 & +1 & 0 & 17,08 & 16,12 \\
5 & 0 & 0 & 95,56 & 65,50 \\
6 & 0 & 0 & 95,56 & 59,46 \\
7 & 0 & 00,32 & 62,48 \\
\hline
\end{tabular}

Os resultados podem ser visualizados nas superfícies de resposta (Figuras 2A e 2B) e nos diagramas de Pareto (Figuras 2C e 2D). Todos os parâmetros estudados mostraram-se significativos para um nível de $95 \%$ de confiança quando utilizada a biomassa de $N$. cochenillifera. Entretanto, o elevado valor do efeito da curvatura mostra mais uma vez a o ajuste insuficiente pelo modelo linear. Merece destaque o elevado rendimento obtido no ponto central (média de 93,81\%) para esta espécie.

Quando utilizada a biomassa de Opuntia, o diagrama de Pareto apresentou resultados semelhantes àqueles obtidos com a cepa CA-11, não tendo a carga inicial de celulose e a interação entre os dois efeitos estudados significância estatística para um nível de confiança de 95\%. Os maiores rendimentos obtidos com ambas as espécies se localizaram no ponto central, o que gerou o elevado efeito da curvatura. Pode-se, portanto, afirmar que a cepa PE-2 demonstrou-se em todos os casos superior à cepa CA-11, em termos de rendimento de conversão da glicose gerada pela hidrólise da celulose em etanol. Nopalea.

A biomassa de Opuntia resultou em todos os casos em rendimentos inferiores aos da palma 


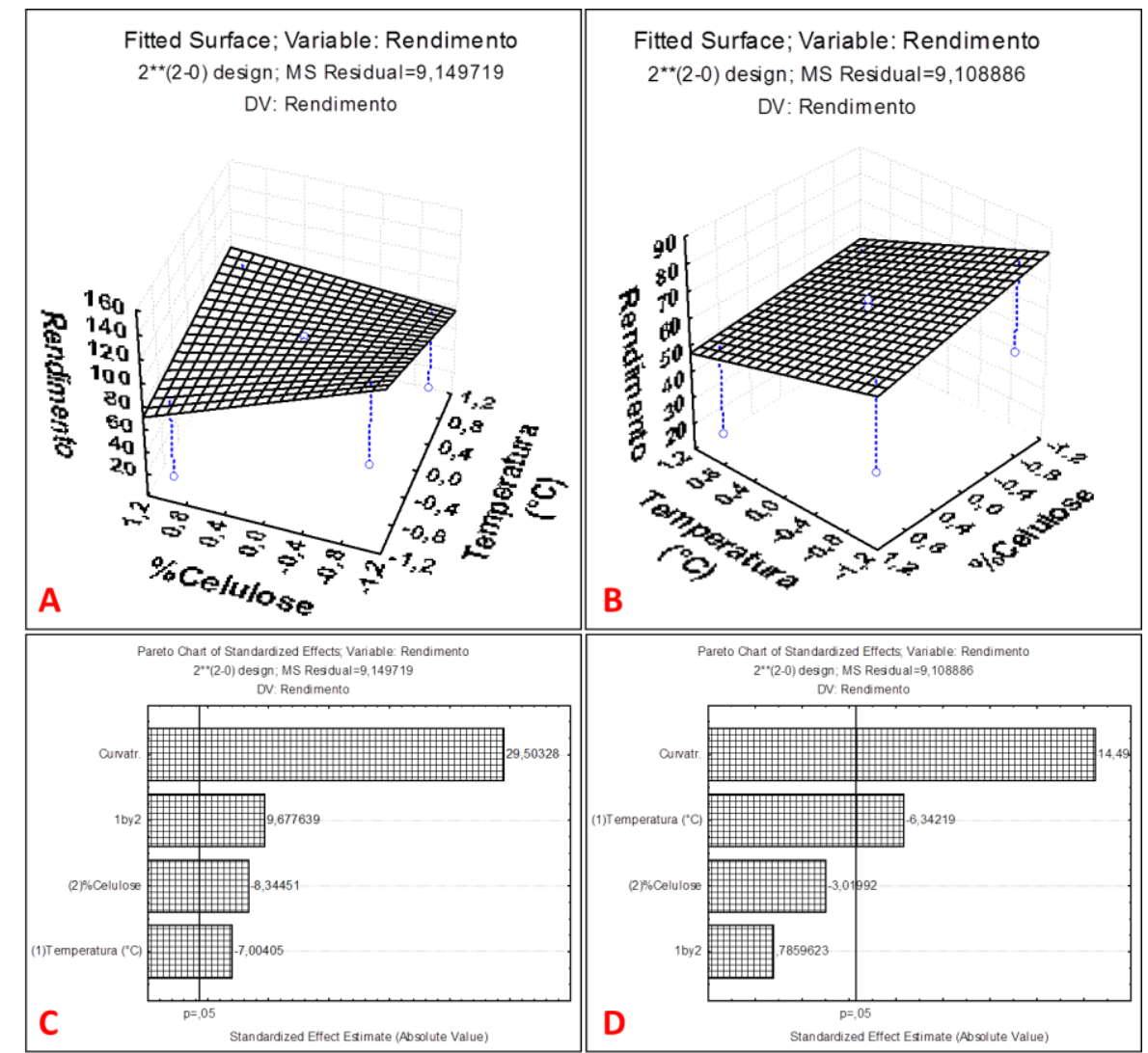

Figura 2 - Curvas de superfície de resposta do processo SFS usando o microrganismo S. cerevisiae, cepa LNF CA-11, e biomassa de $N$. cochenillifera (A) e $O$. ficus indica (B) como substrato e diagramas de Pareto usando $N$. cochenillifera (C) e O. ficus indica (D). Rendimento da conversão de celulose em etanol em função da temperatura e da carga inicial de celulose.

\section{CONCLUSÕES}

A palma forrageira (Opuntia fícus indica e Nopalea cochenillifera) demonstrou-se uma matéria-prima potencial para a produção do etanol de segunda geração, apresentando baixo teor de lignina e concentrações atraentes de polissacarídeos (celulose e hemicelulose). A biomassa da $N$. cochenillifera apresentou altos valores de rendimento de conversão de celulose em etanol pelo processo SFS. Os testes com palma $O$. fícus indica apresentaram valores mais modestos. A cepa PE-2 da levedura Saccharomyces cerevisiae apresentou seus melhores valores de conversão de glicose em etanol na temperatura de $40{ }^{\circ} \mathrm{C}$, enquanto a cepa floculante CA-11 demonstrou melhor desempenho a $35{ }^{\circ} \mathrm{C}$. Não houve efeito significativo do teor de celulose inicial na fermentação nos resultados de conversão, exceto no caso em que se usou a Nopalea e a cepa PE-2.

\section{REFERÊNCIAS}

BARACHO, T. H. A.; SILVA, F. L. H.; TORRES NETO, A. B. Utilização de matérias primas lignocelulósicas para produção de álcool etílico: estudos da hidrólise ácida da palma forrageira 


\section{9 a 22 de outubro de 2014 \\ Florianópolis/SC}

e da destilação do hidrolisado fermentado. In: CONGRESSO BRASILEIRO DE ENGENHARIA QUÍMICA EM INICIAÇÃO CIENTÍFICA, 7, 2009, Uberlândia.

BASSO, L. C.; AMORIM, H. V.; OLIVEIRA, A. J.; LOPES, M. L. Yeast selection for fuel ethanol production in Brazil. FEMS Yeast Research, v. 8, p. 1155-1163, 2008.

DOWE, N.; MCMILLAN, J. SSF Experimental Protocols - Lignocellulosic Biomass Hydrolysis and Fermentation. NREL, Technical Report, 2008.

FOX, J. M.; LEVINE, S. E.; BLANCH, H. W.; CLARK, D. S. An evaluation of cellulose saccharification and fermentation with an engineered Saccharomyces cerevisiae capable of cellobiose and xylose utilization. Biotech. J., v. 7, ed.3, p. 361-373, Março 2012.

KULOYO, O. O. Ethanol production by yeast fermentation of an Opuntia ficus-indica biomass hydrolysate. 2012. 174f. Dissertação (Magister Scientiae) - Faculty of Natural and Agricultural Sciences, Department of Microbial, Biochemical and Food Biotechnology, University of the Free State, Bloemfontein, South Africa.

MALAININE, M. E.; DUFRESNE, A. ; DUPEYRE, D. ; MAHROUZ, M. ; VUONG, R. ; VIGNON, M. R. Structure and morphology of cladodes and spines of Opuntia ficus-indica. Cellulose extraction and characterisation. Carbohydr. Polym., v. 51, p. 77-83, 2003.

PITARELO, A. P. Avaliação da susceptibilidade do bagaço e da palha de cana-de-açúcar à bioconversão via pré-tratamento a vapor e hidrólise enzimática. 2007, 142f. Dissertação (Mestrado em Química) - Universidade Federal do Paraná, Curitiba.

RABELO, S. C.; FONSECA, N. A. A.; ANDRADE, R. R.; MACIEL FILHO, R.; COSTA, A. C. Ethanol production from enzymatic hydrolysis of sugarcane bagasse pretreated with lime and alkaline hydrogen peroxide. Biom. \& Bioen., v. 35, p. 2600-2607, 2011.

RUIZ, H. A.; SILVA, D. P.; RUZENE, D. S.; LIMA, L. F.; VICENTE, A. A.; TEIXEIRA, J. A. Bioethanol production from hydrothermal pretreated wheat straw by a flocculating Saccharomyces cerevisiae strain - Effect of process conditions. Fuel, v. 95, p. 528-536, 2012.

SILVA, C. C. F.; SANTOS, L. C. Palma Forrageira (Opuntia Fícus- Indica Mill) como alternativa na alimentação de ruminantes. R. Electr. de Vet. REDVET, v. VII, n. 10, 2006.

SLUITER, A.; HAMES, B.; RUIZ, R.; SCARLATA, C.; SLUITER, J.; TEMPLETON, D.; CROCKER, D. Determination of structural carbohydrates and lignin in biomass. Golden, CO: National Renewable Energy Laboratory, 2011.

TORRES NETO, A. B. Estudo da pré-hidrólise ácida da palma forrageira (Opuntia ficus-indica Mill). 2010. 47f. Dissertação (Mestrado em Engenharia Agrícola) - Centro de Tecnologia e Recursos Naturais, Universidade Federal de Campina Grande, Campina Grande.

VASCONCELOS, S. M. Pré-tratamento de bagaço de cana-de-açúcar com ácido fosfórico diluído para aplicação em biorrefinarias. 2012. 184f. Tese (Doutorado em Engenharia Química) Programa de Pós-Graduação em Engenharia Química, Universidade Federal de Pernambuco, Recife. 